\title{
Complete blow-up and avalanche formation for a parabolic system with non-simultaneous blow-up
}

\author{
Cristina Brändle * \\ Departamento de Matemáticas, U. Carlos III de Madrid \\ 28911 Leganés, Spain \\ Fernando Quirós ${ }^{\dagger}$ \\ Departamento de Matemáticas, U. Autónoma de Madrid \\ 28049 Madrid, Spain \\ Julio D. Rossi $\ddagger$ \\ IMDEA Matemáticas, C-IX, U. Autónoma de Madrid \\ 28049 Madrid, Spain
}

On leave from Departamento de Matemática, FCEyN, U. de Buenos Aires,

(1428) Buenos Aires, Argentina

\begin{abstract}
We study the possibility of defining a nontrivial continuation after the blow-up time for a system of two heat equations with a nonlinear coupling at the boundary. It turns out that any possible continuation that verify a maximum principle is identically infinity after the blow-up time, that is, both components blow up completely. We also analyze the propagation of the singularity to the whole space, the avalanche, when blow-up is non-simultaneous.
\end{abstract}

\section{Introduction and main results}

We consider solutions $(u, v)$ to two heat equations in the half line, $\mathbb{R}_{+}=(0, \infty)$,

$$
\left\{\begin{array}{l}
u_{t}=u_{x x}, \\
v_{t}=v_{x x},
\end{array} \quad(x, t) \in \mathbb{R}_{+} \times(0, T),\right.
$$

with a nonlinear flux coupling at the boundary

$$
\left\{\begin{array}{l}
-u_{x}(0, t)=u^{p_{11}}(0, t) v^{p_{12}}(0, t), \\
-v_{x}(0, t)=u^{p_{21}}(0, t) v^{p_{22}}(0, t),
\end{array} \quad t \in(0, T) .\right.
$$

*e-mail: cristina.brandle@uc3m.es

${ }^{\dagger}$ e-mail: fernando.quiros@uam.es

${ }^{\ddagger}$ e-mail: jrossi@dm.uba.ar

AMS Subject Classification: 35B60, 35K60, 35K57.

Keywords and phrases: complete blow-up, parabolic system, nonlinear boundary conditions, avalanche. 
The initial data

$$
\left\{\begin{array}{l}
u(x, 0)=u_{0}(x), \\
v(x, 0)=v_{0}(x),
\end{array} \quad x \in \mathbb{R}_{+},\right.
$$

are assumed to be nonnegative, nontrivial, continuous, integrable and bounded. We will also assume that they are compatible with the boundary conditions, so that solutions may be (and will be) understood in a classical sense. In order to have a totally coupled system, we impose the condition $p_{i j}>0$ on the nonlinearities. We also require the monotonicity in time of the solution, $u_{t}, v_{t} \geq 0$. This hypothesis, which is common in the literature, is satisfied if $u_{0}^{\prime \prime} \geq 0$ and $v_{0}^{\prime \prime} \geq 0$.

The time $T$ denotes the maximal existence time for the solution $(u, v)$. If it is infinite we say that the solution is global. If it is finite, we have

$$
\limsup _{t \nearrow T}\left\{\|u(\cdot, t)\|_{\infty}+\|v(\cdot, t)\|_{\infty}\right\}=\infty,
$$

and we say that the solution blows up. There are solutions of (1.1)-(1.3) which blow up if and only if the exponents $p_{i j}$ satisfy any of the following conditions,

$$
p_{11}>1, \quad p_{22}>1, \quad p_{12} p_{21}>\left(1-p_{11}\right)\left(1-p_{22}\right),
$$

see [16]. The study of blow-up due to reaction at the boundary, both for scalar problems and for systems (like the one under consideration here), has attracted a lot of attention in recent years, see for example the surveys [4], [6], [11] and the references therein.

The speed at which blow up takes place (the so called blow-up rate), that can be obtained as in [2], implies that for any $x \neq 0$ there is a constant $K=K(x)$ such that $\sup _{t \in(0, T)}\{|u(x, t)|+|v(x, t)|\} \leq K$. Hence, $(u, v)$ blows up only at the origin. Thus, there may be a nontrivial extension of the solution for times $t>T$ in some weak sense. If such a continuation exists, blow-up is said to be incomplete; otherwise, it is called complete. Complete blow-up was first studied for problems where the nonlinearity occurs in the equation as a reaction term, $u_{t}=u_{x x}+f(u)$, see [1], [9], [10], [11], [12], [13], [18]. For the scalar version of the present problem complete blow-up is proved in [7], see also [17].

Our first aim is to study whether blow-up for problem (1.1)-(1.3) is complete or not. A natural way of obtaining a continuation consists of approximating the reaction nonlinearities in the boundary conditions by a sequence of functions that yield global in time solutions, and then pass to the limit in the approximations. Thus, we solve the heat equations (1.1) with initial data (1.3) and boundary conditions

$$
\left\{\begin{array}{l}
-u_{x}(0, t)=f_{n}^{11}(u(0, t)) f_{n}^{12}(v(0, t)), \quad t>0, \\
-v_{x}(0, t)=f_{n}^{21}(u(0, t)) f_{n}^{22}(v(0, t)),
\end{array}\right.
$$

where

$$
f_{n}^{i j}(s)=\min \left\{s^{p_{i j}}, n^{p_{i j}}\right\},
$$

to obtain a globally defined solution $\left(u_{n}, v_{n}\right)$. Since the coupling functions $f_{n}^{i j}$ increase with $n$, the same is true for $u_{n}$ and $v_{n}$. Hence one may attempt to extend the solution 
after $T$ by taking the limit

$$
\lim _{n \rightarrow \infty} u_{n}=\bar{u}, \quad \lim _{n \rightarrow \infty} v_{n}=\bar{v} .
$$

The extension $(\bar{u}, \bar{v})$ obtained in this way is known in the literature as the proper solution, see [10], [11]. It is a minimal solution in the sense that any solution that satisfies a comparison principle must be above it. Next theorem shows that both components of the proper solution, and hence both components of any other reasonable extension, become infinite after the blow-up time; i.e., blow-up is always complete.

Theorem 1.1 If a solution of (1.1)-(1.3) blows up at a finite time $T$, it blows up completely. More precisely, for all $x \in[0, \infty)$ the proper solution satisfies that

$$
\bar{u}(x, t)=\left\{\begin{array}{ll}
u(x, t), & 0<t<T, \\
\lim _{t \nearrow T} u(x, t), & t=T, \\
\infty, & t>T,
\end{array} \quad \bar{v}(x, t)= \begin{cases}v(x, t), & 0<t<T, \\
\lim _{t \nearrow T} v(x, t), & t=T, \\
\infty, & t>T .\end{cases}\right.
$$

Observe that blow-up is complete for both components even if one of them remains bounded up to the blow-up time, a possibility that is not excluded a priori. Indeed, for certain choices of the parameters $p_{i j}$ there are initial data for which one of the components of the system remains bounded while the other blows up. This phenomenon is commonly denoted as non-simultaneous blow-up. The possibility of non-simultaneous blow-up in nonlinear parabolic systems was first mentioned in [19], and has been studied more thoroughly later in [3], [15], [16] and [20]. For problem (1.1)-(1.3) this possibility was analyzed in [14], [16]: there exist solutions such that $u$ blows up at time $T$ while $v$ remains bounded up to this time if and only if

$$
p_{11}>p_{21}+1 \text {. }
$$

Since blow-up takes place only at one point and there is complete blow-up, at $t=T$ an instantaneous propagation of the blow-up singularity to the whole spatial domain takes place, what is called an avalanche, see [17], [18].

The avalanche may be regarded as a discontinuity at the blow-up time between the nontrivial blow-up profiles

$$
\bar{u}\left(x, T^{-}\right)=\lim _{t \nearrow T} u(x, t) \text { and } \bar{v}\left(x, T^{-}\right)=\lim _{t \nearrow T} v(x, t),
$$

and the trivial values taken afterwards,

$$
\bar{u}\left(x, T^{+}\right)=\infty=\bar{v}\left(x, T^{+}\right) \quad \text { for all } x \in[0, \infty) .
$$

In the case of non-simultaneous blow-up, say $u$ blows up while $v$ remains bounded up to time $T$, the discontinuity at $t=T$ between $\bar{v}\left(x, T^{-}\right.$) (which is finite everywhere) and $\bar{v}\left(x, T^{+}\right)$(which is infinite everywhere) is even more striking. In the sequel we will 
confine ourselves to this more appealing case, assuming that $u$ blows up while $v$ remains bounded up to $t=T^{-}$. In particular, (1.5) holds and we have $p_{11}>1$.

The instantaneous propagation of the singularity to the whole domain has a counterpart when we consider finite, but large, values of $n$ : the propagation of $k$-level sets for $k$ large. Our next aim is to explain the evolution of such level sets. We will devote special attention to $k=n^{\gamma}$. Let us remark that the study of the approximate problems for large $n$ is in many cases (combustion, chemistry) more realistic than the blow-up problem, which is a mathematical idealization.

Let $t_{u}(k)$ be the first time when $u_{n}(0, t)=k$ and $t_{v}(k)$ be the first time when $v_{n}(0, t)=k$. Hence $t_{u}(n)$ and $t_{v}(n)$ are the times at which truncations (1.4) start taking place. In the next result we estimate these times.

Theorem 1.2 Let $n$ be large enough and let $0<\gamma \leq 1$. It holds,

$$
\begin{aligned}
T-t_{u}\left(n^{\gamma}\right) & \sim n^{-2 \gamma\left(p_{11}-1\right)}, \\
t_{v}\left(n^{\gamma}\right)-T & \sim \begin{cases}n^{-2 p_{21}}, & p_{22}>1, \\
n^{-2 p_{21}} \log n, & p_{22}=1, \\
n^{-2 p_{21}+2 \gamma\left(1-p_{22}\right)}, & p_{22}<1 .\end{cases}
\end{aligned}
$$

By $f \sim g$ we mean that there exist constants $c_{1}, c_{2}>0$ such that $c_{1} f \leq g \leq c_{2} f$.

Since $p_{11}>1, t_{u}(n) \nearrow T$ as $n \rightarrow \infty$. However, the behaviour of $t_{v}(n)$ depends on the involved exponents. It may happen that $t_{v}(n) \rightarrow \infty$ as $n \rightarrow \infty$ (this occurs if $\left.p_{21}+p_{22}<1\right)$. This does not contradict the complete blow-up result for $\bar{v}$, since the size of $v_{n}$ for $t>T$ can be very large for $t \approx T$ without reaching the level $n$ before a long time after $T$. In fact, when $p_{22}<1, t_{v}\left(n^{\gamma}\right) \rightarrow T$ as $n \rightarrow \infty$ if and only if $\gamma<p_{21} /\left(1-p_{22}\right)$.

When $p_{22} \geq 1$, the order of magnitude of the quantities $t_{v}\left(n^{\gamma}\right)-T$ does not depend on $\gamma$. Are they equal up to leading order? The answer is given in the next theorem: they are equal when $p_{22}>1$ and differ by a constant coefficient depending on $\gamma$ when $p_{22}=1$.

Theorem 1.3 Let $n$ be large enough and let $0<\gamma \leq 1$. It holds,

(i) If $p_{22}>1$, then $t_{v}(n)-t_{v}\left(n^{\gamma}\right) \sim n^{2\left(\gamma\left(p_{22}-1\right)-p_{21}\right)}$.

(ii) If $p_{22}=1$, then $\lim _{n \rightarrow \infty} \frac{n^{2 p_{21}}}{\log n}\left(t_{v}(n)-t_{v}\left(n^{\gamma}\right)\right)=1-\gamma$.

Next, we want to describe how the level $n$ starts to propagate to the interior of the domain. To this aim, we define

$$
v_{T}=\lim _{t \nearrow T} v(0, t)
$$

and look at times which are close to $t_{u}(n)$, and scale $u_{n}$ by a factor $n$ so that we get something of order one. If we want the new dependent variable to be a solution to the 
heat equation, we also have to scale the space variable. The following result shows how the $n$-level set of $u_{n}$ evolves for times $t \approx T$ close to the origin, $x \approx 0$.

Theorem 1.4 There exists a nontrivial function $\Phi: \mathbb{R}_{+} \times \mathbb{R} \mapsto \mathbb{R}$ such that

$$
\begin{aligned}
& \lim _{n \rightarrow \infty} n^{-1} u_{n}\left(n^{-\left(p_{11}-1\right)} y, t_{u}(n)+n^{-2\left(p_{11}-1\right)} \tau\right)=\Phi(y, \tau), \\
& \lim _{n \rightarrow \infty} v_{n}\left(n^{-\left(p_{11}-1\right)} y, t_{u}(n)+n^{-2\left(p_{11}-1\right)} \tau\right)=v_{T},
\end{aligned}
$$

uniformly on compact subsets of $\mathbb{R}_{+} \times \mathbb{R}$. The limit $\Phi$ depends on the initial data only through $v_{T}$.

Theorem 1.4 implies that the $n$-level set of $u_{n}$ written in terms of $(x, t)$ is given approximately by the 1-level set of the limit function $\Phi$ written in $(y, \tau)$ variables,

$$
\left\{(x, t): u_{n}(x, t)=n\right\} \approx\{(y, \tau): \Phi(y, \tau)=1\} .
$$

These sets are related through the transformation

$$
x=n^{-\left(p_{11}-1\right)} y, \quad t=t_{u}(n)+n^{-2\left(p_{11}-1\right)} \tau .
$$

A monotonicity argument shows that the 1-level set of $\Phi$ can be expressed as the graph of some increasing function $g$. Since $t_{u}(n) \approx T$, this in turn implies that the $n$-level set of $u_{n}$ is given approximately by

$$
\left\{(x, t): x=n^{-\left(p_{11}-1\right)} g\left(n^{2\left(p_{11}-1\right)}(t-T)\right)\right\} .
$$

Remark. The function that describes the onset of the avalanche for $u$ reaches the truncation level 1 at some finite time. Hence, its long time behaviour is given by a self-similar solution of the heat equation in the half-line with inwards flux equal to $v_{T}^{p_{12}}$ at the boundary, i.e.

$$
w(y, \tau) \sim v_{T}^{p_{12}} \tau^{1 / 2} \Phi\left(\frac{y}{\tau^{1 / 2}}\right),
$$

where $\Phi$ solves $\Phi^{\prime \prime}(\xi)+\frac{1}{2} \xi \Phi^{\prime}(\xi)-\frac{1}{2} \Phi(\xi)=0,-\Phi^{\prime}(0)=1$, see [17] for the details. Using this asymptotic behaviour we see that the $k$-level set of $u_{n}$ behaves as

$$
y=\tau^{1 / 2} \Phi^{-1}\left(\frac{k}{v_{T}^{p_{12}} \tau^{1 / 2}}\right) .
$$

We have described the onset of the avalanche for the $u$ variable. However, since the obtained profile for $v$ is the constant $v_{T}$, there is some lack of information concerning the spatial shape of the $v$ variable. To obtain a more precise description of the behaviour of $v_{n}$ we have to look at times which are a little bit larger. 
Theorem 1.5 There exists a nontrivial function $\Psi: \mathbb{R}_{+} \times\left[0, \tau_{0}\right) \mapsto \mathbb{R}$ such that

$$
\lim _{n \rightarrow \infty} v_{n}\left(n^{-p_{21}} y, T+n^{-2 p_{21}} \tau\right)=\Psi(y, \tau)
$$

uniformly on compact subsets of $\mathbb{R}_{+} \times\left[0, \tau_{0}\right)$. The time $\tau_{0}$ verifies $0<\tau_{0}<\infty$ if $p_{22}>1$, $\tau_{0}=\infty$ if $p_{22} \leq 1$. The limit $\Psi$ depends on the initial data only through $v_{T}$.

Remark. If $p_{22}>1$ the limit function $\Psi$ blows up at the threshold time $\tau=\tau_{0}$.

Remark. The fact that $\tau_{0}$ has to be finite for $p_{22}>1$ and not for $p_{22} \leq 1$ could have been guessed from Theorem 1.2.

The behaviour of the $n$-level set of $v_{n}$ in terms of the graph of some increasing function follows arguing as we did before for $u_{n}$.

Theorems 1.4 and 1.5 provide information near the origin close to the blow-up time. To completely describe the avalanche, we also give the behaviour of both components, $u_{n}$ and $v_{n}$, for fixed $(x, t)$, with $t>T$.

Theorem 1.6 Let

$$
\begin{array}{lll}
\gamma_{1}=p_{11}+p_{12}, & \gamma_{2}=p_{21}+p_{22} & \text { if } p_{21}+p_{22}>1, \\
\gamma_{1}=p_{11}+\frac{p_{12} p_{21}}{1-p_{22}}, & \gamma_{2}=\frac{p_{21}}{1-p_{22}} & \text { if } p_{21}+p_{22} \leq 1 .
\end{array}
$$

There exist profiles $\varphi$ and $\psi$ such that

$$
\lim _{n \rightarrow \infty} n^{-\gamma_{1}} u_{n}(x, t)=\varphi(x, t), \quad \lim _{n \rightarrow \infty} n^{-\gamma_{2}} v_{n}(x, t)=\psi(x, t),
$$

uniformly on compact subsets of $[0, \infty) \times(T, \infty)$. The limits $\varphi, \psi$ are independent of the initial data.

The quantity $p_{21}+p_{22}$ measures the combined strength of the two reaction factors in the boundary condition for $v$. If it is above 1 , reaction is big, and $v_{n}$ reaches the $n$-level quickly, in a time $t_{v}(n) \rightarrow T$ as $n \rightarrow \infty$. Hence, for any time $t>T$ all truncations have taken place for $n$ large. Thus, $u_{n}$ and $v_{n}$ are both solutions of the heat equation with inwards boundary fluxes given respectively by $n^{p_{11}+p_{12}}$ and $n^{p_{21}+p_{22}}$. What the theorem says is that we have to scale precisely by these powers of $n$ if we want to obtain a non-trivial limit. Consider now $p_{21}+p_{22}<1$. Given any fixed time $t$, the truncation of $v_{n}$ takes place afterwards for all $n$ large enough. In this case the exponent $\gamma_{2}$ which gives the size of $v_{n}$ coincides with the critical value of $\gamma$ below which $t_{v}\left(n^{\gamma}\right)$ goes to $T$. The adequate value of $\gamma_{1}$ follows by inserting the size of $v_{n}$ in the boundary condition for $u_{n}$. In any case, the scaling exponents $\gamma_{1}, \gamma_{2}$ are the only ones such that $t_{u}\left(n^{\gamma_{1}}\right)$, $t_{v}\left(n^{\gamma_{2}}\right)$ do not go neither to $T$ nor to infinity, see Section 3 .

Our last step consists in considering the asymptotic behaviour of $u_{n}$ and $v_{n}$ as $t \rightarrow \infty$. Since we are considering $t>t_{v}(n)$, both truncations have taken place. Again, $u_{n}$ and 
$v_{n}$ are solutions of the heat equation with inwards boundary fluxes given respectively by $n^{p_{11}+p_{12}}$ and $n^{p_{21}+p_{22}}$. As is well known, solutions to this problem with integrable initial datum converge, uniformly on compact subsets, as $t$ goes to infinity to the solution of the same problem with zero initial data, see for instance [17]. This solution is self-similar, and coincides with the limit functions of Theorem 1.6 in the case $p_{21}+p_{22}>1$.

Remark. The nontrivial limit profiles that appear in Theorems 1.4, 1.5, and 1.6 are solutions of explicit problems, see Sections 4 and 5.

Organization of the PAPER. In Section 2 we prove complete blow-up for both components, Theorem 1.1. The estimates for $t_{u}\left(n^{\gamma}\right)-T$ and $t_{v}\left(n^{\gamma}\right)-T$ that give us the right scalings to describe the avalanche are gathered in Section 3. It includes both the case $\gamma \leq 1$, Theorem 1.2, and the case $\gamma>1$. In order to deal with this latter case we have to study the size of $u_{n}$ at time $t_{v}(n)$, a result that is interesting on its own. Section 4 is devoted to prove Theorems 1.4 and 1.5, which describe the onset of the avalanche. Finally, in Section 5, we prove Theorem 1.6.

Throughout the paper $C, c$ denote constants, independent of $n$, which may be different in different occurrences.

\section{Complete blow-up}

Proof of Theorem 1.1. We can assume without loss of generality that $u$ blows up at time $t=T$. If this not the case, then $v$ has to blow up, and the same proof applies interchanging the roles of $u$ and $v$. The component $v$ may blow up or not, since we have not assumed a priori that blow-up is non-simultaneous.

For times before the blow-up time, $t<T,(u, v)$ is bounded. Hence, if $n$ is large enough, $(u, v)$ solves the truncated problem up to time $t$ and therefore $u_{n}(x, t)=u(x, t)$ and $v_{n}(x, t)=v(x, t)$.

To study what happens exactly at $t=T$ we use the monotonicity with respect to $t$ and a comparison argument. For $t<T$ we have

$$
\lim _{t \nearrow T} u(x, t) \geq u_{n}(x, T) \geq u_{n}(x, t) .
$$

Taking limits as $n \rightarrow \infty$, and using that $\lim _{n \rightarrow \infty} u_{n}(x, t)=u(x, t)$ for $t<T$, and then letting $t \nearrow T$, we get

$$
\lim _{t \nearrow T} u(x, t) \geq \lim _{n \rightarrow \infty} u_{n}(x, T) \geq \lim _{t \nearrow T} u(x, t) .
$$

The same applies to $v$.

To end the proof we have to study the behaviour for $t>T$. From the previous step we know that $\lim _{t \nearrow T} u(0, t)=\lim _{n \rightarrow \infty} u_{n}(0, T)$. Since $u_{n}$ is increasing in time and $u$ blows up at the origin,

$$
\infty=\lim _{t \nearrow T} u(0, t)=\lim _{n \rightarrow \infty} u_{n}(0, T) \leq \lim _{n \rightarrow \infty} u_{n}(0, t)
$$


and we conclude that the proper solution, $\bar{u}=\lim _{n \rightarrow \infty} u_{n}$, is identically infinite at the origin for $t>T$. In order to propagate the singularity to the whole interval for both components we use the representation formula obtained from the heat kernel. Let $\Gamma$ be the fundamental solution of the heat equation in $\mathbb{R}_{+} \times(0, \infty)$, namely

$$
\Gamma(x, t)=\frac{1}{(\pi t)^{1 / 2}} \exp \left(-\frac{x^{2}}{4 t}\right) .
$$

For $x \in \mathbb{R}_{+}$we have

$$
\begin{aligned}
u_{n}(x, t)= & \int_{\mathbb{R}_{+}} u_{n}(y, 0) \Gamma(x-y, t) d y-\int_{0}^{t} \frac{\partial u_{n}}{\partial x}(0, \tau) \Gamma(x, t-\tau) d \tau \\
& -\int_{0}^{t} u_{n}(0, \tau) \frac{\partial \Gamma}{\partial x}(x, t-\tau) d \tau .
\end{aligned}
$$

Since $\Gamma$ and the boundary flux, $-\left(u_{n}\right)_{x}(0, \tau)$, are both nonnegative we can bound $u_{n}(x, t)$ from below by

$$
u_{n}(x, t) \geq-\int_{0}^{t} u_{n}(0, \tau) \frac{\partial \Gamma}{\partial x}(x, t-\tau) d \tau
$$

From (2.1) we have that $u_{n}(0, t) \geq M$ if $n$ is large enough, how large depending on $M$. Hence, for any $0<\delta<t-T$,

$$
u_{n}(x, t) \geq M \int_{T}^{T+\delta}\left(-\frac{\partial \Gamma}{\partial x}(x, t-\tau)\right) d \tau .
$$

We conclude that

$$
\lim _{n \rightarrow \infty} u_{n}(x, t)=\infty, \quad t>T, x \in \mathbb{R}_{+} .
$$

This proves complete blow-up for $u$.

To obtain complete blow-up for $v$ we also use the representation formula. Since $v_{n}$ grows due to the influence of $u_{n}$ through the boundary flux, we keep the flux term in the bound from below:

$$
v_{n}(x, t) \geq \int_{0}^{t} f_{n}^{21}\left(u_{n}(0, \tau)\right) f_{n}^{22}\left(v_{n}(0, \tau)\right) \Gamma(x, t-\tau) d \tau .
$$

From the monotonicity of the solutions we have that $v_{n}(0, t) \geq v_{n}(0,0)=v(0,0)=c>0$. Therefore, if $n$ is large enough and for any $0<\delta<t-T$,

$$
v_{n}(x, t) \geq M^{p_{21}} c^{p_{22}} \int_{T}^{T+\delta} \Gamma(x, t-\tau) d \tau .
$$

This implies complete blow-up for $v$, that is,

$$
\lim _{n \rightarrow \infty} v_{n}(x, t)=\infty, \quad t>T, x \in \mathbb{R}_{+} .
$$




\section{Time estimates}

The aim of this section is to find estimates for the differences $t_{u}\left(n^{\gamma}\right)-T$ and $t_{v}\left(n^{\gamma}\right)-T$ for different values of $\gamma$.

As blow-up is non-simultaneous, the $u$ component touches the level $n$ before the $v$ component, which is bounded up to time $T$. Since the solutions of the truncated problems coincide with those of the non-truncated one until some component reaches the level $n$, we have $t_{u}(n)<T<t_{v}\left(n^{\gamma}\right)$ for any $\gamma>0$ and large $n$. If $\gamma>1, t_{u}\left(n^{\gamma}\right)>t_{u}(n)$. However, we cannot state at this point whether $t_{u}\left(n^{\gamma}\right)$ lies before or after $T$. The aim of this section is to estimate the times $t_{u}\left(n^{\gamma}\right)$ and $t_{v}\left(n^{\gamma}\right)$ for any $\gamma>0$. In order to compute $t_{u}\left(n^{\gamma}\right)$ for $\gamma>1$ we need to estimate the size of $u_{n}$ at time $t_{v}(n)$, a result that is interesting on its own.

In the sequel we use the notation

$$
\alpha=\frac{p_{21}}{\left(p_{22}-1\right)}, \quad \beta=\frac{1}{2\left(p_{22}-1\right)}
$$

for a couple of exponents that will appear frequently.

\subsection{Estimates for $0<\gamma \leq 1$}

Proof of Theorem 1.2. We first obtain an estimate for $t_{u}\left(n^{\gamma}\right)$, which will then be used to estimate $t_{v}\left(n^{\gamma}\right)$.

Estimates for $t_{u}\left(n^{\gamma}\right)$. Since $t_{u}\left(n^{\gamma}\right) \leq t_{u}(n)<T$ for $\gamma \leq 1$, from the blow-up rate of $u$, which is known to be, see [8],

$$
\max _{x} u(x, t)=u(0, t) \sim(T-t)^{-\frac{1}{2\left(p_{11}-1\right)}},
$$

we can obtain the estimate for $t_{u}\left(n^{\gamma}\right)$. Indeed, from (3.1) we get

$$
n^{\gamma} \sim\left(T-t_{u}\left(n^{\gamma}\right)\right)^{-\frac{1}{2\left(p_{11}-1\right)}},
$$

which is equivalent to (1.6).

Estimates for $t_{v}\left(n^{\gamma}\right)$. We look at the problem satisfied by $v_{n}$ for times larger than $t_{u}(n)$. For these times the nonlinearity that involves the $u$ variable is truncated. Hence, $v_{n}$ is a solution to

$$
\begin{cases}\left(v_{n}\right)_{t}=\left(v_{n}\right)_{x x}, & (x, t) \in \mathbb{R}_{+} \times\left(t_{u}(n), \infty\right), \\ -\left(v_{n}\right)_{x}(0, t)=n^{p_{21}} f_{n}^{22}\left(v_{n}(0, t)\right), & t \in\left(t_{u}(n), \infty\right), \\ v_{n}\left(x, t_{u}(n)\right)=v\left(x, t_{u}(n)\right), & x \in \mathbb{R}_{+} .\end{cases}
$$

Since blow-up is non-simultaneous, $v$ is bounded up to time $T$. Hence the initial datum in (3.2) is also bounded, $v\left(x, t_{u}(n)\right)<v(x, T)$. The idea is comparing $v_{n}$ with a selfsimilar solution $z$ which has an explicit formula, so that $t_{z}\left(n^{\gamma}\right)$, the first time when $z$ reaches level $n^{\gamma}$, can be computed. 
$p_{22}>1$ The function $z(x, t)=n^{-\alpha}(\widehat{T}-t)^{-\beta} \phi\left(x(\widehat{T}-t)^{-1 / 2}\right)$, is a self-similar solution to (3.2) for times at which $f_{n}^{22}(z)=z^{p_{22}}$, i.e., for $t \leq t_{z}(n)$. See [8] for the existence of the profile $\phi$, which has an explicit formula.

If we take $n^{-\alpha} \widehat{T}^{-\beta} \leq C$, then $z(x, 0)=n^{-\alpha} \widehat{T}^{-\beta} \phi\left(x \widehat{T}^{-1 / 2}\right) \leq v_{n}\left(x, t_{u}(n)\right)$. Using a comparison argument it follows that

$$
z(x, t) \leq v_{n}\left(x, t+t_{u}(n)\right), \quad 0<t<t_{z}(n) .
$$

Since $\gamma \leq 1, t_{z}\left(n^{\gamma}\right) \leq t_{z}(n)$, and hence

$$
t_{v}\left(n^{\gamma}\right) \leq t_{u}(n)+t_{z}\left(n^{\gamma}\right)
$$

But we have an explicit estimate for $t_{z}\left(n^{\gamma}\right)$ :

$$
z\left(0, t_{z}\left(n^{\gamma}\right)\right)=n^{\gamma}=n^{-\alpha}\left(\widehat{T}-t_{z}\left(n^{\gamma}\right)\right)^{-\beta} \phi(0) .
$$

Hence

$$
\widehat{T}-t_{z}\left(n^{\gamma}\right) \sim n^{-2\left(\gamma\left(p_{22}-1\right)+p_{21}\right)},
$$

and therefore, using that $\widehat{T} \leq C n^{-2 p_{21}}=C n^{-2 p_{21}}$, we get

$$
t_{z}\left(n^{\gamma}\right) \leq-C n^{-2\left(\gamma\left(p_{22}-1\right)+p_{21}\right)}+C n^{-2 p_{21}} \leq C n^{-2 p_{21}} .
$$

From (3.3) using the non-simultaneous blow-up condition on the exponents, $p_{21}<p_{11}-1$, we obtain,

$$
t_{v}\left(n^{\gamma}\right)-T \leq t_{u}(n)-T+t_{z}\left(n^{\gamma}\right) \leq-C n^{-2\left(p_{11}-1\right)}+C n^{-2 p_{21}} \leq C n^{-2 p_{21}} .
$$

On the other hand, if we now choose $C$, independent of $n$, so that $n^{-\alpha} \widehat{T}^{-\beta} \geq C$, we get $z(x, 0) \geq v_{n}\left(x, t_{u}(n)\right)$ and hence

$$
z(x, t) \geq v_{n}\left(x, t+t_{u}(n)\right), \quad t>t_{z}(n) .
$$

Using the same idea we get $t_{v}\left(n^{\gamma}\right)-T \geq c n^{-2 p_{21}}$, and conclude that $t_{v}\left(n^{\gamma}\right)-T \sim n^{-2 p_{21}}$. $p_{22}<1$ The same argument can be carried over considering the self-similar solution $z(x, t)=n^{-\alpha}(\widehat{T}+t)^{-\beta} \phi\left(x(\widehat{T}+t)^{-1 / 2}\right)$. The main difference between this and the previous case is that now, since $p_{22}<1$, the estimate (3.5) becomes

$$
t_{z}\left(n^{\gamma}\right) \leq-C n^{-2\left(\gamma\left(p_{22}-1\right)+p_{21}\right)}+C n^{-2 p_{21}} \leq C n^{-2\left(\gamma\left(p_{22}-1\right)+p_{21}\right)},
$$

respectively $\geq$. In this case, we conclude that

$$
t_{v}\left(n^{\gamma}\right)-T \sim-n^{2\left(p_{11}-1\right)}+n^{2\left(1-p_{22}\right) \gamma-2 p_{21}} \sim n^{2\left(1-p_{22}\right) \gamma-2 p_{21}} .
$$

$p_{22}=1$ In this critical case, if we look for a self-similar solution as before, $\alpha$ and $\beta$ are not well defined and hence we take as self-similar solution of $(3.2), z(x, t)=K e^{a t-b x}$, 
with $a=b^{2}=n^{2 p_{21}}$ and $K$ a constant. Arguing as before, taking $K$ small or big, so that $z(x, 0) \sim v_{n}\left(x, t_{u}(n)\right)$, we obtain $t_{v}\left(n^{\gamma}\right)-T \sim n^{-2 p_{21}} \log n$.

Remark. As a byproduct of this proof we have obtained that

$$
v\left(x, t+t_{u}(n)\right) \sim z(x, t),
$$

for times $0<t<t_{z}(n) \sim t_{v}(n)-t_{u}(n)$, with $z$ the self-similar solution defined before according to the sign of $p_{22}-1$. We also obtain that

$$
t_{z}(n) \sim t_{v}(n)-t_{u}(n) \sim \begin{cases}n^{-2 p_{21}}, & p_{22}>1 \\ n^{-2 p_{21}+2\left(1-p_{22}\right)}, & p_{22}<1\end{cases}
$$

and for $p_{22}=1$ a better estimate

$$
e^{n^{2 p_{21} t_{z}(n)}} \sim e^{n^{2 p_{21}\left(t_{v}(n)-t_{u}(n)\right)} \sim n}
$$

Remark. Using (3.3) and (3.4) we get $\widehat{T}-\left(t_{v}(n)-t_{u}(n)\right) \geq c\left(\widehat{T}-t_{z}(n)\right)$. An analogous computation proves the reverse inequality. We conclude that

$$
\widehat{T}-\left(t_{v}(n)-t_{u}(n)\right) \sim \widehat{T}-t_{z}(n) \sim n^{-2\left(p_{22}-1\right)-2 p_{21}} .
$$

Proof of Theorem 1.3. We use the same technique used in the proof of Theorem 1.2, but taking as initial time $t_{v}\left(n^{\gamma}\right)$. Let us sketch it briefly for $p_{22}>1: v_{n}$ is a solution of

$$
\begin{cases}\left(v_{n}\right)_{t}=\left(v_{n}\right)_{x x}, & (x, t) \in \mathbb{R}_{+} \times\left(t_{v}(n), \infty\right), \\ -\left(v_{n}\right)_{x}(0, t)=n^{p_{21}} f_{n}^{22}\left(v_{n}(0, t)\right), & t \in\left(t_{v}\left(n^{\gamma}\right), \infty\right), \\ v_{n}\left(x, t_{v}\left(n^{\gamma}\right)\right)=n^{\gamma}, & x \in \mathbb{R}_{+} .\end{cases}
$$

If we take $n^{-\alpha} \widehat{T}^{-\beta} \sim n^{\gamma}$, then $z(x, 0)=n^{-\alpha} \widehat{T}^{-\beta} \phi\left(x \widehat{T}^{-1 / 2}\right) \sim v_{n}\left(x, t_{v}\left(n^{\gamma}\right)\right)$, and hence, by comparison

$$
t_{v}(n) \sim t_{v}\left(n^{\gamma}\right)+t_{z}(n) .
$$

Since $\widehat{T}-t_{z}(n) \sim n^{-\frac{1+\alpha}{\beta}}$, we conclude, using that $\gamma<1$, that $t_{z}(n) \sim n^{-\frac{\gamma+\alpha}{\beta}}$ and therefore from (3.11), $t_{v}(n)-t_{v}\left(n^{\gamma}\right) \sim n^{-\frac{\gamma+\alpha}{\beta}}$.

\subsection{Sizes}

Next theorem gives an estimate of the size of $u_{n}$ at time $t_{v}(n)$.

Theorem 3.1 The size of $u_{n}$ at time $t_{v}(n)$ is

$$
u_{n}\left(0, t_{v}(n)\right) \sim \begin{cases}n^{p_{11}-p_{21}+p_{12}+1-p_{22},} & p_{22}<1+p_{12}, \\ n^{p_{11}-p_{21}} \log n, & p_{22}=1+p_{12}, \\ n^{p_{11}-p_{21}}, & p_{22}>1+p_{12}\end{cases}
$$


Proof. Let $t_{u}(n)<t<t_{v}(n)$ and $d(n)=t_{v}(n)-t_{u}(n)$. For those times we have that $u_{n}$ is a solution of the heat equation with boundary and initial data given by

$$
\left\{\begin{array}{l}
-\left(u_{n}\right)_{x}(0, t) \sim n^{p_{11}} v_{n}^{p_{12}}(0, t) \\
u_{n}\left(x, t_{u}(n)\right) \sim n
\end{array}\right.
$$

Consider $\widehat{u}$ a solution to the heat equation in $\mathbb{R}_{+} \times(0, \infty)$ with

$$
\left\{\begin{array}{l}
-\widehat{u}_{x}(0, t)=n^{p_{11}} v_{n}^{p_{12}}\left(0, t+t_{u}(n)\right) \\
\widehat{u}(x, 0)=n
\end{array}\right.
$$

A comparison argument gives that $u_{n}\left(x, t+t_{u}(n)\right) \sim \widehat{u}(x, t)$.

Using formula $(2.2)$ we can estimate $\widehat{u}(0, t)$ at $d(n)$. Indeed,

$$
\begin{aligned}
\widehat{u}(0, d(n)) & =\int_{\mathbb{R}_{+}} \widehat{u}(y, d(n)) \Gamma(y, d(n)) d y+\int_{0}^{d(n)} n^{p_{11}} v_{n}^{p_{12}}\left(0, \tau+t_{u}(n)\right) \Gamma(0, d(n)-\tau) d \tau \\
& \sim n+n^{p_{11}} \int_{0}^{d(n)} v_{n}^{p_{12}}(0, \tau)(d(n)-\tau)^{-1 / 2} d \tau=n+n^{p_{11}} I .
\end{aligned}
$$

Hence, our aim is estimating $I$ according to the estimates we have for $v_{n}$, see (3.6), in order to get the size of $u_{n}$ at $t_{v}(n)$.

$p_{22}<1$ Using (3.6) for $0<t<t_{v}(n)-t_{u}(n)$, we have $v_{n}\left(0, t+t_{u}(n)\right) \sim n^{-\alpha}(\widehat{T}+t)^{-\beta}$. Hence,

$$
\begin{aligned}
I \sim & \int_{0}^{d(n)} n^{-\alpha p_{12}}(\widehat{T}+\tau)^{-\beta p_{12}}(d(n)-\tau)^{-1 / 2} d \tau \\
= & n^{-\alpha p_{12}} \int_{0}^{d(n)}(\widehat{T}+\tau)^{-\beta p_{12}}(d(n)-\tau)^{-1 / 2} d \tau \\
\leq & C n^{-\alpha p_{12}}\left(d(n)^{-1 / 2} \int_{0}^{d(n) / 2}(\widehat{T}+\tau)^{-\beta p_{12}} d \tau\right. \\
& \left.\quad+(\widehat{T}+d(n))^{-\beta p_{12}} \int_{d(n) / 2}^{d(n)}(d(n)-\tau)^{-1 / 2} d \tau\right) \\
& \leq C n^{-\alpha p_{12}}\left(d(n)^{-1 / 2}(d(n)+\widehat{T})^{-\beta p_{12}+1}+(\widehat{T}+d(n))^{-\beta p_{12}} d(n)^{1 / 2}\right) \\
\leq & C n^{-\alpha p_{12}} d(n)^{-\beta p_{12}+1 / 2},
\end{aligned}
$$

where we have used that $d(n) \sim n^{-2 p_{21}+2\left(1-p_{22}\right)}>\widehat{T} \sim n^{-2 p_{21}}$, see (3.7). On the other hand,

$$
\begin{aligned}
I & \sim \int_{0}^{d(n)} n^{-\alpha p_{12}}(\widehat{T}+\tau)^{-\beta p_{12}}(d(n)-\tau)^{-1 / 2} d \tau \\
& \geq n^{-\alpha p_{12}}(\widehat{T}+d(n))^{-\beta p_{12}} \int_{0}^{d(n)}(d(n)-\tau)^{-1 / 2} \geq C n^{-\alpha p_{12}} d(n)^{-\beta p_{12}+1 / 2} .
\end{aligned}
$$


We conclude, using again (3.7), that

$$
\widehat{u}\left(0, t_{v}(n)-t_{u}(n)\right) \sim n+n^{p_{11}} n^{-\alpha p_{12}} n^{-2\left(p_{21}+p_{22}-1\right)\left(-\beta p_{12}+1 / 2\right)},
$$

and hence $u_{n}\left(0, t_{v}(n)\right) \sim n+n^{p_{11}-p_{21}+p_{12}+1-p_{22}} \sim n^{p_{11}-p_{21}+p_{12}+1-p_{22}}$.

$p_{22}=1 \quad$ According to $(3.6)$ we set $v_{n}\left(x, t+t_{u}(n)\right) \sim K e^{a t-b x}$, so that

$$
\begin{aligned}
I & \sim \int_{0}^{d(n)} e^{a p_{12} \tau}(d(n)-\tau)^{-1 / 2} d \tau \\
& \geq e^{a p_{12}(d(n)-d(n) / \log n)} \int_{d(n)-d(n) / \log n}^{d(n)}(d(n)-\tau)^{-1 / 2} d \tau \geq C n^{p_{12}} n^{p_{21}},
\end{aligned}
$$

where we use that $d(n) \sim n^{2 p_{21}} \log (n)$ and estimate (3.8).

Following the idea of the case $p_{22}<1$, we split $I$ into two integrals that are treated in a different way, in order to obtain a bound from above,

$$
\begin{aligned}
& I \sim \int_{0}^{d(n)} e^{a p_{12} \tau}(d(n)-\tau)^{-1 / 2} d \tau \\
& =\int_{0}^{d(n)-d(n) / \log n} e^{a p_{12} \tau}(d(n)-\tau)^{-1 / 2} d \tau \\
& \quad \quad+\int_{d(n)-d(n) / \log n}^{d(n)} e^{a p_{12} \tau}(d(n)-\tau)^{-1 / 2} d \tau \\
& \leq n^{p_{21}} \int_{0}^{d(n)-d(n) / \log n} e^{a p_{12} \tau} d \tau \\
& \quad+n^{p_{12}} \int_{d(n)-d(n) / \log n}^{d(n)}(d(n)-\tau)^{-1 / 2} d \tau \leq C n^{p_{12}-p_{21}} .
\end{aligned}
$$

We conclude that $u\left(0, t_{v}(n)\right) \sim n^{p_{11}+p_{12}-p_{21}}$.

$1<p_{22}<1+p_{12}$ As $p_{22}>1$, using again (3.6) we get $v_{n}\left(0, t+t_{u}(n)\right) \sim n^{-\alpha}(\widehat{T}-t)^{-\beta}$.

In order to find a bound from above for $\widehat{u}$ we observe that similar computations as the ones done in the case $p_{22}<1$ do not yield a "reasonable" bound, since we obtain $\widehat{u} \leq \infty$. Hence, instead of using the representation formula, we compare $\widehat{u}$ with

$$
U(x, t)=n^{-\lambda}(\widehat{T}-t)^{-\mu} G\left(x(\widehat{T}-t)^{-1 / 2}\right),
$$

which is a solution to

$$
\left\{\begin{array}{l}
U_{t}=U_{x x}, \\
-U_{x}(0, t)=n^{p_{11}-\alpha p_{12}}(\widehat{T}-t)^{-\beta p_{12}} G^{\prime}(0), \\
U(x, 0)=n^{p_{11}-p_{21}} G\left(x \widehat{T}^{-1 / 2}\right),
\end{array}\right.
$$

if $\mu=\beta p_{12}-1 / 2$ and $\lambda=\alpha p_{12}-p_{11}$. Due to the assumption of non-simultaneous blow-up we have that $p_{11}-p_{21}>1$, hence $U(x, 0)>n=\widehat{u}(x, 0)$ and a comparison argument yields $U(0, d(n)) \geq \widehat{u}(0, d(n))$. Hence, using (3.9), we conclude that

$$
\widehat{u}(0, d(n)) \leq C n^{p_{11}-\alpha p_{12}}(\widehat{T}-d(n))^{-\mu} \leq C n^{p_{12}-p_{22}+1-p_{21}+p_{11}} .
$$


In order to obtain the lower bound we observe that $-\beta p_{12}+1 / 2<0$ and hence, since $\widehat{T}>d(n)$,

$$
\begin{aligned}
I & \sim n^{-\alpha p_{12}} \int_{0}^{d(n)}(\widehat{T}-\tau)^{-\beta p_{12}}(d(n)-\tau)^{-1 / 2} d \tau \\
& =n^{-\alpha p_{12}} \int_{0}^{d(n)}(\widehat{T}-\tau)^{-\beta p_{12}-1 / 2} \frac{(\widehat{T}-\tau)^{1 / 2}}{(d(n)-\tau)^{1 / 2}} d \tau \\
& \geq C n^{-\alpha p_{12}}\left((\widehat{T}-d(n))^{-\beta p_{12}+1 / 2}-\widehat{T}^{-\beta p_{12}+1 / 2}\right) \\
& \geq C n^{-\alpha p_{12}}\left((\widehat{T}-d(n))^{-\beta p_{12}+1 / 2}\left(1-\frac{\widehat{T}^{-\beta p_{12}+1 / 2}}{(\widehat{T}-d(n))^{-\beta p_{12}+1 / 2}}\right)\right. \\
& \geq C n^{-\alpha p_{12}}(\widehat{T}-d(n))^{-\beta p_{12}+1 / 2} \geq C n^{p_{12}-p_{22}+1-p_{21} .}
\end{aligned}
$$

Summing up, $u_{n}\left(0, t_{v}(n)\right) \sim n+n^{p_{11}-p_{21}+p_{12}-p_{22}+1} \sim n^{p_{11}-p_{21}+p_{12}-p_{22}+1}$.

$p_{22}=1+p_{12} \quad$ In this critical case, we compute $I$ explicitly and obtain

$$
I \sim n^{-\alpha p_{12}} \int_{0}^{d(n)}(\widehat{T}-\tau)^{-1 / 2}(d(n)-\tau)^{-1 / 2} d \tau=n^{-\alpha p_{12}} \log \left|\frac{d(n)-\widehat{T}}{2 \sqrt{\widehat{T} d(n)}-\widehat{T}-d(n)}\right| .
$$

But,

$$
\left|\frac{d(n)-\widehat{T}}{2 \sqrt{\widehat{T} d(n)}-\widehat{T}-d(n)}\right|=\frac{\widehat{T}-d(n)}{\widehat{T}\left(1-\sqrt{\frac{d(n)}{\hat{T}}}\right)^{2}} .
$$

Since $d(n)<\widehat{T}$ and $\widehat{T}-d(n) \sim n^{-2\left(p_{12}-p_{21}\right)}$, see (3.9), we conclude that $I \sim n^{-\alpha p_{12}} \log n$, which yields $u_{n}\left(0, t_{v}(n)\right) \sim n^{p_{11}-\alpha p_{12}} \log n$.

$p_{22}>1+p_{12} \quad$ Although $p_{22}>1$, and hence $v_{n}\left(0, t+t_{u}(n)\right) \sim n^{-\alpha}(\widehat{T}-t)^{-\beta}$, the main difference between this case and the case $1<p_{22}<1+p_{12}$ is that now $-\beta p_{12}+1 / 2>0$. Thus, the estimates for $I$ are done in a different way:

$$
\begin{aligned}
I & \sim n^{-\alpha p_{12}} \int_{0}^{d(n)}(\widehat{T}-\tau)^{-\beta p_{12}}(d(n)-\tau)^{-1 / 2} d \tau \leq n^{-\alpha p_{12}} \int_{0}^{d(n)}(d(n)-\tau)^{-\beta p_{12}-1 / 2} \\
& \leq C n^{-\alpha p_{12}} d(n)^{-\beta p_{12}+1 / 2} .
\end{aligned}
$$

On the other hand,

$$
\begin{aligned}
I & \sim n^{-\alpha p_{12}} \int_{0}^{d(n)}(\widehat{T}-\tau)^{-\beta p_{12}}(d(n)-\tau)^{-1 / 2} d \tau \\
& =n^{-\alpha p_{12}} \int_{0}^{d(n)}(\widehat{T}-\tau)^{-\beta p_{12}-1 / 2} \frac{(\widehat{T}-\tau)^{1 / 2}}{(d(n)-\tau)^{1 / 2}} d \tau \\
& \geq C n^{-\alpha p_{12}}\left(\widehat{T}^{-\beta p_{12}+1 / 2}-(\widehat{T}-d(n))^{-\beta p_{12}+1 / 2}\right) \geq C n^{-\alpha p_{12}} \widehat{T}^{-\beta p_{12}+1 / 2} .
\end{aligned}
$$

Hence, since $\widehat{T} \sim d(n)$, we get $I \sim n^{-\alpha p_{12}} d(n)^{-\beta p_{12}+1 / 2}$, and we conclude using (3.7) that $u_{n}\left(0, t_{v}(n)\right) \sim n+n^{p_{11}-p_{21}} \sim n^{p_{11}-p_{21}}$. 


\subsection{Estimates for $\gamma>1$}

Estimates for $t_{v}\left(n^{\gamma}\right)$. Since $t_{v}\left(n^{\gamma}\right)-T=t_{v}\left(n^{\gamma}\right)-t_{v}(n)+t_{v}(n)-T$, we can use (1.7) with $\gamma=1$, so that it only remains to compute $t_{v}\left(n^{\gamma}\right)-t_{v}(n)$.

Theorem 3.2 Let $n$ be large enough and $\gamma>1$. It holds,

$$
t_{v}\left(n^{\gamma}\right)-T \sim \begin{cases}n^{-2 p_{21}}, & 1<\gamma \leq p_{22}, \\ n^{2\left(\gamma-\left(p_{21}+p_{22}\right)\right)}, & \gamma>p_{22} .\end{cases}
$$

Proof. For times $t>t_{v}(n)$ the truncations have taken place; hence $v_{n}$ is a solution of the heat equation with boundary data $-\left(v_{n}\right)_{x}(0, t)=n^{p_{21}+p_{22}}$, and initial data $v_{n}\left(x, t_{v}(n)\right) \sim n$.

Using the representation formula for the heat equation, (2.2), we get that

$$
\begin{aligned}
v_{n}\left(0, t_{v}\left(n^{\gamma}\right)\right)= & \int_{\mathbb{R}_{+}} v_{n}\left(y, t_{v}(n)\right) \Gamma\left(y, t_{v}\left(n^{\gamma}\right)-t_{v}(n)\right) d y \\
& \left.+\int_{0}^{t_{v}\left(n^{\gamma}\right)-t_{v}(n)} n^{p_{21}+p_{22}} \Gamma\left(0, t_{v}\left(n^{\gamma}\right)-t_{v}(n)\right)-\tau\right) d \tau \\
& -\int_{0}^{t_{v}\left(n^{\gamma}\right)-t_{v}(n)} v_{n}(0, \tau) \frac{\partial \Gamma}{\partial x}\left(0, t_{v}\left(n^{\gamma}\right)-t_{v}(n)-\tau\right) d \tau \\
\sim & n+n^{p_{21}+p_{22}} \int_{0}^{t_{v}\left(n^{\gamma}\right)-t_{v}(n)} \Gamma\left(0, t_{v}\left(n^{\gamma}\right)-t_{v}(n)-\tau\right) d \tau \\
= & n+C n^{p_{21}+p_{22}}\left(t_{v}\left(n^{\gamma}\right)-t_{v}(n)\right)^{1 / 2} .
\end{aligned}
$$

Since $v_{n}\left(0, t_{v}\left(n^{\gamma}\right)\right)=n^{\gamma}$ and $\gamma>1$ we get

$$
t_{v}\left(n^{\gamma}\right)-t_{v}(n) \sim n^{2\left(\gamma-\left(p_{21}+p_{22}\right)\right)} .
$$

This expression together with (1.7) yields (3.13).

Estimates for $t_{u}\left(n^{\gamma}\right)$. The idea is to use the estimates of the size of $u_{n}(0, t)$ at $t=t_{v}(n)$, that we have just obtained, to see if it has reached or not the level $n^{\gamma}$. If the size of $u_{n}$ at $t_{v}(n)$ is smaller than $n^{\gamma}$, then $t_{u}\left(n^{\gamma}\right)>t_{v}(n)$, and we estimate the difference $t_{u}\left(n^{\gamma}\right)-T$ taking into account the size of $u_{n}\left(x, t_{v}(n)\right)$ and that for $t>t_{v}(n)$ both truncations have taken place. If the size of $u_{n}$ at $t_{v}(n)$ is bigger than $n^{\gamma}$, we use that $u_{n}$ is a solution of the heat equation, with boundary flux $-\left(u_{n}\right)_{x}(0, t) \sim n^{p_{11}} v^{p_{12}}(0, t)$, and initial data $u\left(x, t_{u}(n)\right) \sim n$. If $u_{n}\left(0, t_{v}(n)\right)=n^{\gamma}$, then $t_{u}\left(n^{\gamma}\right)=t_{v}(n)$ and the result follows from (1.7). In the critical cases $\gamma=p_{11}-p_{21}, p_{11}-p_{21}+p_{12}-p_{22}+1$, the size estimates do not imply an ordering between $t_{u}\left(n^{\gamma}\right)$ and $t_{v}(n)$; we will have to take into account the two possibilities separately. 

have

If $\gamma<\max \left\{p_{11}-p_{21}, p_{11}-p_{21}+p_{12}-p_{22}+1\right\}$, which implies $t_{u}\left(n^{\gamma}\right)<t_{v}(n)$, we

$$
\begin{aligned}
n^{\gamma}=u_{n}\left(0, t_{u}\left(n^{\gamma}\right)\right) & \sim \widehat{u}\left(0, t_{u}\left(n^{\gamma}\right)-t_{u}(n)\right) \\
& \sim n+n^{p_{11}} \int_{0}^{t_{u}\left(n^{\gamma}\right)-t_{u}(n)} v^{p_{12}}\left(0, \tau+t_{u}(n)\right)\left(t_{u}\left(n^{\gamma}\right)-t_{u}(n)-\tau\right)^{-\frac{1}{2}} d \tau \\
& =n+n^{p_{11}} \mathcal{I},
\end{aligned}
$$

and since $\gamma>1$ we get

$$
\mathcal{I} \sim n^{\gamma-p_{11}} .
$$

Let us sketch briefly how to bound $\mathcal{I}$. As before, $t_{u}\left(n^{\gamma}\right)-T=t_{u}\left(n^{\gamma}\right)-t_{u}(n)+t_{u}(n)-T$, so that we only have to estimate $t_{u}\left(n^{\gamma}\right)-t_{u}(n)$, (remember that $T-t_{u}(n) \sim n^{-2\left(p_{11}-1\right)}$, see (1.6)). We follow the proof of Theorem 3.1, replacing $d(n)$ by $t_{u}\left(n^{\gamma}\right)-t_{u}(n)$. Note that, since $t_{v}(n)>t_{u}\left(n^{\gamma}\right)$, we have that $d(n)>t_{u}\left(n^{\gamma}\right)-t_{u}(n)$.

Theorem 3.3 Let $n$ be large enough and let $1<\gamma<p_{11}-p_{21}$. It holds,

$$
t_{u}\left(n^{\gamma}\right)-T \sim n^{-2\left(p_{11}-\gamma\right)} .
$$

Proof. The main difference between this proof and that of Theorem 3.1 is that we do not know the relation between $t_{u}\left(n^{\gamma}\right)-t_{u}(n)$ and $\widehat{T}$.

$p_{22}<1 \quad$ Assume first that $t_{u}\left(n^{\gamma}\right)-t_{u}(n)>\widehat{T}$, then

$$
\begin{aligned}
\mathcal{I} & \sim n^{-\alpha p_{12}} \int_{0}^{t_{u}\left(n^{\gamma}\right)-t_{u}(n)}(\widehat{T}+\tau)^{-\beta p_{12}}\left(t_{u}\left(n^{\gamma}\right)-t_{u}(n)-\tau\right)^{-1 / 2} d \tau \\
& \sim n^{-\alpha p_{12}}\left(t_{u}\left(n^{\gamma}\right)-t_{u}(n)\right)^{-\beta p_{12}+1 / 2} .
\end{aligned}
$$

Hence, using (3.14), $t_{u}\left(n^{\gamma}\right)-t_{u}(n) \sim n^{\frac{\gamma-p_{11}+\alpha p_{12}}{-\beta p_{12}+1 / 2}}$. Thus, if $\gamma<p_{11}-p_{21}$ then

$$
\frac{\gamma-p_{11}+\alpha p_{12}}{-\beta p_{12}+1 / 2}<-2 p_{21} \text {. }
$$

Since $\widehat{T} \sim n^{-2 p_{21}}$ this means that $t_{u}\left(n^{\gamma}\right)-t_{u}(n)<\widehat{T}$ and therefore, in this range of values for $\gamma$, we can not have $t_{u}\left(n^{\gamma}\right)-t_{u}(n)>\widehat{T}$. In other words, the region where $t_{u}\left(n^{\gamma}\right)-t_{u}(n)>\widehat{T}$ is at most $p_{11}-p_{21}+p_{12}-p_{22}+1>\gamma>p_{11}-p_{21}$, and in this region we get

$$
t_{u}\left(n^{\gamma}\right)-T \sim n^{\frac{\gamma-p_{11}-\alpha p_{12}}{-\beta p_{12}+1 / 2}}-\left(T-t_{u}(n)\right) \sim n^{\frac{\gamma-p_{11}+\alpha p_{12}}{-\beta p_{12}+1 / 2}}-n^{-2\left(p_{11}-1\right)} \sim n^{\frac{\gamma-p_{11}+\alpha p_{12}}{-\beta p_{12}+1 / 2}} .
$$

On the other hand, if $t_{u}\left(n^{\gamma}\right)-t_{u}(n)<\widehat{T}$, then

$$
\begin{aligned}
\mathcal{I} & \sim n^{-\alpha p_{12}} \int_{0}^{t_{u}\left(n^{\gamma}\right)-t_{u}(n)}(\widehat{T}+\tau)^{-\beta p_{12}}\left(t_{u}\left(n^{\gamma}\right)-t_{u}(n)-\tau\right)^{-1 / 2} d \tau \\
& \leq C n^{-\alpha p_{12}} \widehat{T}^{-\beta p_{12}} \int_{0}^{t_{u}\left(n^{\gamma}\right)-t_{u}(n)}\left(t_{u}\left(n^{\gamma}\right)-t_{u}(n)-\tau\right)^{-1 / 2} d \tau \\
& \leq C n^{-\alpha p_{12}} \widehat{T}^{-\beta p_{12}}\left(t_{u}\left(n^{\gamma}\right)-t_{u}(n)\right)^{1 / 2} .
\end{aligned}
$$


For the upper bound we have

$$
\begin{aligned}
\mathcal{I} & \sim n^{-\alpha p_{12}} \int_{0}^{t_{u}\left(n^{\gamma}\right)-t_{u}(n)}(\widehat{T}+\tau)^{-\beta p_{12}}\left(t_{u}\left(n^{\gamma}\right)-t_{u}(n)-\tau\right)^{-1 / 2} d \tau \\
& \geq n^{-\alpha p_{12}}(\widehat{T}+d(n))^{-\beta p_{12}} \int_{0}^{d(n)}\left(t_{u}\left(n^{\gamma}\right)-t_{u}(n)-\tau\right)^{-1 / 2} \\
& \geq C n^{-\alpha p_{12}}(\widehat{T}+d(n))^{-\beta p_{12}}\left(t_{u}\left(n^{\gamma}\right)-t_{u}(n)\right)^{1 / 2} \geq C n^{-\alpha p_{12}} \widehat{T}^{-\beta p_{12}}\left(t_{u}\left(n^{\gamma}\right)-t_{u}(n)\right)^{1 / 2} .
\end{aligned}
$$

Hence, $t_{u}\left(n^{\gamma}\right)-t_{u}(n) \sim n^{2\left(\gamma-p_{11}\right)}$. As before, we conclude that the values of $\gamma$ for which $t_{u}\left(n^{\gamma}\right)-t_{u}(n)<\widehat{T}$ is a subset of $1<\gamma<p_{11}-p_{21}$, where we have

$$
t_{u}\left(n^{\gamma}\right)-T \sim n^{2\left(\gamma-p_{11}\right)}-\left(T-t_{u}(n)\right) \sim n^{2\left(\gamma-p_{11}\right)}-n^{-2\left(p_{11}-1\right)} \sim n^{2\left(\gamma-p_{11}\right)} .
$$

Summing up

$$
t_{u}\left(n^{\gamma}\right)-T \sim \begin{cases}n^{\frac{\gamma-p_{11}+\alpha p_{12}}{-\beta p_{12}+1 / 2}}, & \gamma>p_{11}-p_{21} \\ n^{2\left(\gamma-p_{11}\right)}, & \gamma<p_{11}-p_{21}\end{cases}
$$

$p_{22}=1 \quad$ We bound $\mathcal{I}$ from above in the following way:

$$
\begin{aligned}
\mathcal{I} & \sim \int_{0}^{t_{u}\left(n^{\gamma}\right)-t_{u}(n)} e^{a p_{12} \tau}\left(t_{u}\left(n^{\gamma}\right)-t_{u}(n)-\tau\right)^{-1 / 2} d \tau \\
& \geq \int_{0}^{t_{u}\left(n^{\gamma}\right)-t_{u}(n)}\left(t_{u}\left(n^{\gamma}\right)-t_{u}(n)-\tau\right)^{-1 / 2} d \tau \geq C\left(t_{u}\left(n^{\gamma}\right)-t_{u}(n)\right)^{1 / 2} .
\end{aligned}
$$

Hence

$$
t_{u}\left(n^{\gamma}\right)-t_{u}(n) \leq C n^{2\left(\gamma-p_{11}\right)}
$$

On the other hand,

$$
\begin{aligned}
\mathcal{I} & \sim \int_{0}^{t_{u}\left(n^{\gamma}\right)-t_{u}(n)} e^{a p_{12} \tau}\left(t_{u}\left(n^{\gamma}\right)-t_{u}(n)-\tau\right)^{-1 / 2} d \tau \\
& \leq e^{p_{12} a\left(t_{u}\left(n^{\gamma}\right)-t_{u}(n)\right)} \int_{0}^{t_{u}\left(n^{\gamma}\right)-t_{u}(n)}\left(t_{u}\left(n^{\gamma}\right)-t_{u}(n)-\tau\right)^{-1 / 2} d \tau .
\end{aligned}
$$

Inequality (3.18) and the fact that $\gamma<p_{11}-p_{21}$ imply that $a\left(t_{u}\left(n^{\gamma}\right)-t_{u}(n)\right) \leq C$. Hence, $t_{u}\left(n^{\gamma}\right)-t_{u}(n) \geq n^{2\left(\gamma-p_{11}\right)}$. It follows that $t_{u}\left(n^{\gamma}\right)-t_{u}(n) \sim n^{2\left(\gamma-p_{11}\right)}$, which yields (3.15).

$p_{22}>1$ The idea is the same as in the case $p_{22}=1$, we first bound $t_{u}\left(n^{\gamma}\right)-t_{u}(n)$ from above and then use this estimate to obtain the lower bound,

$$
\begin{aligned}
\mathcal{I} & \sim n^{-\alpha p_{12}} \int_{0}^{t_{u}\left(n^{\gamma}\right)-t_{u}(n)}(\widehat{T}-\tau)^{-\beta p_{12}}\left(t_{u}\left(n^{\gamma}\right)-t_{u}(n)-\tau\right)^{-1 / 2} d \tau \\
& \geq C n^{-\alpha p_{12}} \widehat{T}^{-\beta p_{12}} \int_{0}^{t_{u}\left(n^{\gamma}\right)-t_{u}(n)}\left(t_{u}\left(n^{\gamma}\right)-t_{u}(n)-\tau\right)^{-1 / 2} d \tau \\
& \geq C n^{-\alpha p_{12}} \widehat{T}^{-\beta p_{12}}\left(t_{u}\left(n^{\gamma}\right)-t_{u}(n)\right)^{1 / 2} .
\end{aligned}
$$


Hence

$$
t_{u}\left(n^{\gamma}\right)-t_{u}(n) \leq C n^{2\left(\gamma-p_{11}\right)}
$$

On the other hand,

$$
\begin{aligned}
\mathcal{I} & \sim n^{-\alpha p_{12}} \int_{0}^{t_{u}\left(n^{\gamma}\right)-t_{u}(n)}(\widehat{T}-\tau)^{-\beta p_{12}}\left(t_{u}\left(n^{\gamma}\right)-t_{u}(n)-\tau\right)^{-1 / 2} d \tau \\
& \leq n^{-\alpha p_{12}}\left(\widehat{T}-\left(t_{u}\left(n^{\gamma}\right)-t_{u}(n)\right)\right)^{-\beta p_{12}} \int_{0}^{t_{u}\left(n^{\gamma}\right)-t_{u}(n)}\left(t_{u}\left(n^{\gamma}\right)-t_{u}(n)-\tau\right)^{-1 / 2} d \tau \\
& \leq C n^{-\alpha p_{12}}\left(\widehat{T}-\left(t_{u}\left(n^{\gamma}\right)-t_{u}(n)\right)\right)^{-\beta p_{12}}\left(t_{u}\left(n^{\gamma}\right)-t_{u}(n)\right)^{1 / 2} .
\end{aligned}
$$

By (3.19), we have that $\widehat{T} \sim n^{-2 p_{21}}>t_{u}\left(n^{\gamma}\right)-t_{u}(n)$. Therefore,

$$
\mathcal{I} \leq C n^{-\alpha p_{12}} \widehat{T}^{-\beta p_{12}}\left(t_{u}\left(n^{\gamma}\right)-t_{u}(n)\right)^{1 / 2} .
$$

We conclude again that $t_{u}\left(n^{\gamma}\right)-t_{u}(n) \sim n^{2\left(\gamma-p_{11}\right)}$.

Theorem 3.4 Let $n$ be large enough and let $p_{11}-p_{21}<\gamma<p_{11}-p_{21}+p_{12}-p_{22}+1$. It holds,

$$
t_{u}\left(n^{\gamma}\right)-T \sim \begin{cases}n^{\frac{\gamma-p_{11}+\alpha p_{12}}{-\beta p_{12}+1 / 2}}, & p_{22}<1, \\ n^{-2 p_{21}} \log n, & p_{22}=1, \\ n^{-2 p_{21}}, & 1<p_{22}<1+p_{12} .\end{cases}
$$

Remark. In the range $p_{11}-p_{21}<\gamma<p_{11}-p_{21}+p_{12}-p_{22}+1$, we have $p_{22}<1+p_{12}$.

Proof. $p_{22}<1 \quad$ See the proof of Theorem 3.3, formulas (3.16) and (3.17).

$p_{22}=1 \quad$ In this case, since $\gamma>p_{11}-p_{21}$, inequality (3.18) does not imply that $t_{u}\left(n^{\gamma}\right)-t_{u}(n)<n^{-2 p_{21}}$, and we have to find a bound for $\mathcal{I}$ in a different way:

$$
\begin{aligned}
\mathcal{I} & \geq e^{a p_{12}\left(t_{u}\left(n^{\gamma}\right)-t_{u}(n)\right)} \int_{t_{u}\left(n^{\gamma}\right)-t_{u}(n)-d(n) / \log n}^{t_{u}\left(n^{\gamma}\right)-t_{u}(n)}\left(\left(t_{u}\left(n^{\gamma}\right)-t_{u}(n)\right)-\tau\right)^{-1 / 2} d \tau \\
& \geq C n^{-p_{21}} e^{p_{12} n^{2 p_{21}}\left(t_{u}\left(n^{\gamma}\right)-t_{u}(n)\right)} .
\end{aligned}
$$

Following the proof of Theorem 3.1, we split $\mathcal{I}$ into two integrals,

$$
\begin{aligned}
\mathcal{I} \sim & \int_{0}^{t_{u}\left(n^{\gamma}\right)-t_{u}(n)-d(n) / \log n} e^{a p_{12} \tau}\left(t_{u}\left(n^{\gamma}\right)-t_{u}(n)-\tau\right)^{-1 / 2} d \tau \\
& +\int_{t_{u}\left(n^{\gamma}\right)-t_{u}(n)-d(n) / \log n}^{t_{u}\left(n^{\gamma}\right)-t_{u}(n)} e^{a p_{12} \tau}\left(t_{u}\left(n^{\gamma}\right)-t_{u}(n)-\tau\right)^{-1 / 2} d \tau \\
\leq & n^{p_{21}} \int_{0}^{t_{u}\left(n^{\gamma}\right)-t_{u}(n)-d(n) / \log n} e^{a p_{12} \tau} d \tau \\
& +e^{a p_{12}\left(t_{u}\left(n^{\gamma}\right)-t_{u}(n)\right)} \int_{t_{u}\left(n^{\gamma}\right)-t_{u}(n)-d(n) / \log n}^{t_{u}\left(n^{\gamma}\right)-t_{u}(n)}\left(t_{u}\left(n^{\gamma}\right)-t_{u}(n)-\tau\right)^{-1 / 2} d \tau \\
\leq & C n^{-p_{21}} e^{p_{12} n^{2 p_{21}}\left(t_{u}\left(n^{\gamma}\right)-t_{u}(n)\right)} .
\end{aligned}
$$


We conclude that

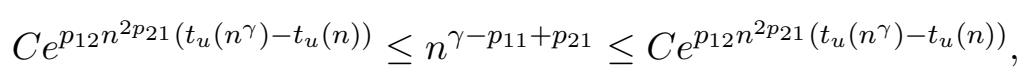

and hence, since $\gamma>p_{11}-p_{21}$,

$$
C\left(\gamma-p_{11}+p_{21}\right) n^{-2 p_{21}} \log n \leq t_{u}\left(n^{\gamma}\right)-t_{u}(n) \leq\left(\gamma-p_{11}+p_{21}\right) n^{-2 p_{21}} \log n .
$$

Therefore, using (1.6), $t_{u}\left(n^{\gamma}\right)-T \sim\left(\gamma-p_{11}+p_{21}\right) n^{-2 p_{21}} \log n$.

$1<p_{22}<1+p_{12} \quad$ Comparison with the self-similar solution (3.12) yields

$$
n^{\gamma}=\widehat{u}\left(0, t_{u}\left(n^{\gamma}\right)-t_{u}(n)\right) \leq n^{p_{11}-\alpha p_{12}}\left(\widehat{T}-\left(t_{u}\left(n^{\gamma}\right)-t_{u}(n)\right)\right)^{-\beta p_{12}+1 / 2} .
$$

On the other hand,

$$
\begin{aligned}
\mathcal{I} & \geq n^{-\alpha p_{12}} \int_{0}^{t_{u}\left(n^{\gamma}\right)-t_{u}(n)}(\widehat{T}-\tau)^{-\beta p_{12}-1 / 2} \frac{(\widehat{T}-\tau)^{1 / 2}}{\left(t_{u}\left(n^{\gamma}\right)-t_{u}(n)-\tau\right)^{1 / 2}} d \tau \\
& \geq n^{-\alpha p_{12}}\left(\widehat{T}-\left(t_{u}\left(n^{\gamma}\right)-t_{u}(n)\right)\right)^{-\beta p_{12}+1 / 2}
\end{aligned}
$$

Therefore,

$$
\widehat{T}-n^{\frac{\gamma-p_{11}+\alpha p_{12}}{-\beta p_{12}+1 / 2}} \leq t_{u}\left(n^{\gamma}\right)-t_{u}(n) \leq \widehat{T}-n^{\frac{\gamma-p_{11}+\alpha p_{12}}{-\beta p_{12}+1 / 2}} .
$$

The fact that $\gamma>p_{11}-p_{21}$ implies that $t_{u}\left(n^{\gamma}\right)-t_{u}(n) \sim \widehat{T} \sim n^{-2 p_{21}}$, from where the Theorem follows.

We consider now $\gamma>\max \left\{p_{11}-p_{21}, p_{11}-p_{21}+p_{12}-p_{22}+1\right\}$. We have that $t_{u}\left(n^{\gamma}\right)>t_{v}(n)$ and hence $u_{n}$ satisfies the heat equation in $\left(t_{v}(n), \infty\right)$ with boundary flux $-\left(u_{n}\right)_{x}(0, t) \sim n^{p_{11}+p_{12}}$. The initial data is the size of $u_{n}$ at $t_{v}(n)$; i.e, $u_{n}\left(x, t_{v}(n)\right) \sim n^{\eta}$, with $\eta=\max \left\{p_{11}-p_{21}, p_{11}-p_{21}+p_{12}-p_{22}+1\right\}$, see Theorem 3.1.

Theorem 3.5 Let $n$ be large enough and let $\gamma>\max \left\{p_{11}-p_{21}, p_{11}-p_{21}+p_{12}-p_{22}+1\right\}$. It holds,

$$
t_{u}\left(n^{\gamma}\right)-T \sim \begin{cases}n^{-2 p_{21}}, & \gamma \leq p_{11}+p_{12}-p_{21} \\ n^{2\left(\gamma-p_{11}-p_{12}\right)}, & \gamma>p_{11}+p_{12}-p_{21}\end{cases}
$$

Proof. Using the representation formula,

$$
\begin{aligned}
n^{\gamma}=\widehat{u}\left(0, t_{u}\left(n^{\gamma}\right)-t_{v}(n)\right) & \sim n^{\eta}+n^{p_{11}+p_{12}} \int_{0}^{t_{u}\left(n^{\gamma}\right)-t_{v}(n)}\left(t_{u}\left(n^{\gamma}\right)-t_{v}(n)-\tau\right)^{-1 / 2} d \tau \\
& \sim n^{\eta}+n^{p_{11}+p_{12}}\left(t_{u}\left(n^{\gamma}\right)-t_{v}(n)\right)^{1 / 2} .
\end{aligned}
$$

Since $\gamma>\eta$ we get $t_{u}\left(n^{\gamma}\right)-t_{v}(n) \sim n^{2\left(\gamma-p_{11}-p_{12}\right)}$; hence, using see (1.7), (3.20) follows.

Finally, we consider the critical cases.

Theorem 3.6 Let $n$ be large enough. 
(i) If $\gamma=p_{11}-p_{21}$, then $t_{u}\left(n^{\gamma}\right)-T \sim n^{-2 p_{21}}$.

(ii) If $\gamma=p_{11}-p_{21}+p_{12}-p_{22}+1$, then

$$
t_{u}\left(n^{\gamma}\right)-T \sim \begin{cases}n^{2\left(-p_{21}+1-p_{22}\right)}, & p_{22}<1 \\ n^{-2 p_{21}} \log n, & p_{22}=1 \\ n^{-2 p_{21}}, & p_{22}>1\end{cases}
$$

Proof. Assume first that $t_{u}\left(n^{\gamma}\right)<t_{v}(n)$. If $\gamma=p_{11}-p_{21}$, then the proof of Theorem 3.3 yields $t_{u}\left(n^{\gamma}\right)-t_{u}(n) \sim n^{-2 p_{21}}$. In the case $p_{22}>1$ we have to use that $\widehat{T}>t_{u}\left(n^{\gamma}\right)-t_{u}(n)$, which follows from $\widehat{T}>t_{v}(n)-t_{u}(n)$. If $\gamma=p_{11}-p_{21}+p_{12}-p_{22}+1$ the proof of Theorem 3.4 can extended to include this case.

If $t_{u}\left(n^{\gamma}\right)>t_{v}(n)$ the proof of Theorem 3.5 still applies. Notice that when $\gamma=\eta$, though $u_{n}\left(0, t_{v}(n)\right) \sim n^{\gamma}$, we still have $(3.20)$, since $u_{n}\left(0, t_{v}(n)\right)<n^{\gamma}$.

We summarize the results of this subsection in Figure 1. It is worth noticing that the order of magnitude of $t_{u}\left(n^{\gamma}\right)-T$ depends in a continuous manner on the parameters except across the segment $p_{22}=1, p_{11}-p_{21}<\gamma \leq p_{11}-p_{21}+p_{12}$, where it appears a logarithmic correction.

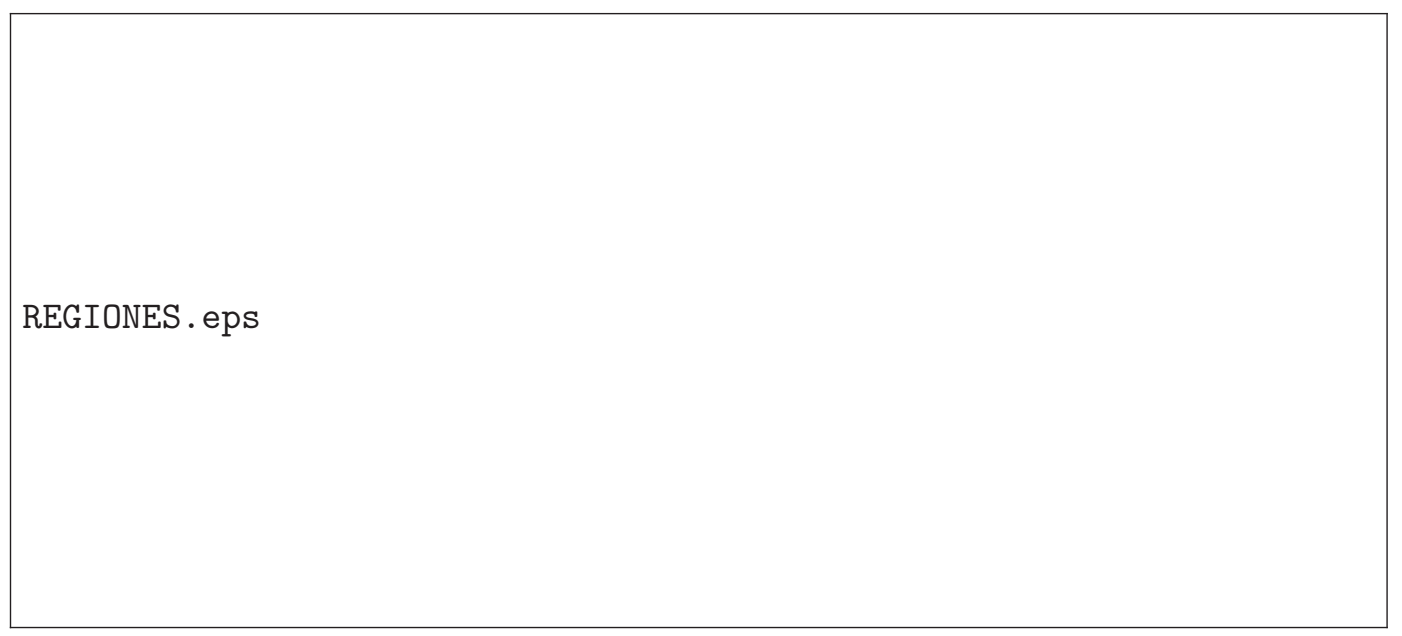

Figure 1: $\quad t_{u}\left(n^{\gamma}\right)-T$

\section{Onset of the avalanche}

The aim of this section is to prove theorems 1.4 and 1.5. In order to study the onset of the avalanche for the component $u$ we first need to know its behaviour at time $t=T^{-}$. It turns out that it is given by a self-similar profile. 
Lemma 4.1 Let $p>1$ and let $u$ be a solution to

$$
\begin{cases}u_{t}=u_{x x}, & (x, t) \in \mathbb{R}_{+} \times(0, T), \\ -u_{x}(0, t)=g(t) u^{p}(0, t), & t \in(0, T), \\ u(x, 0)=u_{0}(x), & x \in \mathbb{R}_{+},\end{cases}
$$

where $g$ is monotone increasing and bounded and $u$ blows up at time T. Then

$$
\lim _{t \nearrow T}(T-t)^{\frac{1}{2(p-1)}} u\left(y(T-t)^{1 / 2}, t\right)=g(T)^{\frac{1}{p-1}} \zeta(y),
$$

uniformly on compact intervals $0 \leq y \leq C$, where

$$
\zeta(y)=\frac{1}{\sqrt{\pi} \Gamma\left(\frac{1}{2(p-1)}\right)}\left(\frac{\Gamma\left(\frac{p}{2(p-1)}\right)}{2(p-1) \Gamma\left(\frac{2 p-1}{2(p-1)}\right)}\right)^{\frac{1}{p-1}} \int_{0}^{\infty} e^{-\frac{y^{2}}{4 t}} \frac{3-2 p}{2(p-1)}^{(1+t)^{-\frac{p}{2(p-1)}}} d t .
$$

Proof. The blow-up rate is given by

$$
c(T-t)^{\frac{-1}{2(p-1)}} \leq\|u(\cdot, t)\|_{\infty} \leq C(T-t)^{\frac{-1}{2(p-1)}},
$$

see [2], [16]. If we write the function $u$ in self-similar variables we get that the rescaled function

$$
w(y, \tau)=(T-t)^{\frac{1}{2(p-1)}} u\left(y(T-t)^{1 / 2}, t\right), \quad \tau=-\log (T-t),
$$

is a bounded solution of

$$
\begin{cases}w_{\tau}=w_{y y}-\frac{1}{2} y w_{y}-\frac{1}{2(p-1)} w, & (y, \tau) \in \mathbb{R}_{+} \times(-\log T, \infty), \\ -w_{y}(0, \tau)=g\left(T-e^{-\tau}\right) w^{p}(0, \tau), & t \in(-\log T, \infty), \\ w(y,-\log T)=T^{\frac{1}{2(p-1)}} u_{0}\left(y T^{1 / 2}\right), & y \in \mathbb{R}_{+} .\end{cases}
$$

If $g$ is a constant, $g=k$, then $w$ converges uniformly on sets of the form $0 \leq y \leq C$ to a steady state, which is a multiple of the profile $\zeta$,

$$
\lim _{\tau \rightarrow \infty} w(y, \tau)=k^{\frac{1}{p-1}} \zeta(y)
$$

see [8]. For a general monotone $g$ a comparison argument yields that for every $\eta>0$

$$
(g(T-\eta))^{\frac{1}{p-1}} \zeta(y) \leq \liminf _{\tau \rightarrow \infty} w(y, \tau) \leq \limsup _{\tau \rightarrow \infty} w(y, \tau) \leq(g(T))^{\frac{1}{p-1}} \zeta(y)
$$

from where (4.1) follows.

Proof of Theorem 1.4. Since $u_{n}\left(0, t_{u}(n)\right)=n$ and $v_{n}\left(0, t_{u}(n)\right) \leq K$, the change of variables,

$$
\begin{aligned}
& w_{n}(y, \tau)=n^{-1} u_{n}\left(n^{-\left(p_{11}-1\right)} y, t_{u}(n)+n^{-2\left(p_{11}-1\right)} \tau\right), \\
& z_{n}(y, \tau)=v_{n}\left(n^{-\left(p_{11}-1\right)} y, t_{u}(n)+n^{-2\left(p_{11}-1\right)} \tau\right),
\end{aligned}
$$


scales both components to order one for $\tau=0$. We obtain the system

$$
\left\{\begin{array}{l}
\left(w_{n}\right)_{\tau}=\left(w_{n}\right)_{y y}, \\
\left(z_{n}\right)_{\tau}=\left(z_{n}\right)_{y y},
\end{array} \quad(y, \tau) \in \mathbb{R}_{+} \times\left(-n^{2\left(p_{11}-1\right)} t_{u}(n), \infty\right),\right.
$$

coupled with the boundary conditions,

$$
\left\{\begin{array}{l}
-\left(w_{n}\right)_{y}(0, \tau)=f_{1}^{11}\left(w_{n}(0, \tau)\right) f_{n}^{12}\left(z_{n}(0, \tau)\right), \\
-\left(z_{n}\right)_{y}(0, \tau)=n^{-p_{11}+p_{21}} f_{1}^{21}\left(w_{n}(0, \tau)\right) f_{n}^{22}\left(z_{n}(0, \tau)\right),
\end{array} \quad \tau \in\left(-n^{2\left(p_{11}-1\right)} t_{u}(n), \infty\right),\right.
$$

and initial data

$$
\left\{\begin{array}{l}
w_{n}(y, 0)=n^{-1} u_{n}\left(n^{-\left(p_{11}-1\right)} y, t_{u}(n)\right), \quad y \in \mathbb{R}_{+} . \\
z_{n}(y, 0)=v_{n}\left(n^{-\left(p_{11}-1\right)} y, t_{u}(n)\right)
\end{array}\right.
$$

The same super and subsolutions used in the the proof of Theorem 1.2 guarantee that $w_{n}$ and $z_{n}$ are uniformly bounded (independently of $n$ ) in compact subsets of $(y, \tau)$. Therefore, by standard regularity theory, we get uniform bounds in $C^{2+\epsilon, 1+\epsilon / 2}$. Hence, we can pass to the limit as $n \rightarrow \infty$. Let

$$
z(y, \tau)=\lim _{n \rightarrow \infty} z_{n}(y, \tau) .
$$

For any $\tau \leq 0$, we get that $z(y, \tau)=v_{T}$. If $\tau \geq 0$, passing to the limit in the equation for $z_{n}$, we obtain that the limit (uniform on compact subsets), $z$, satisfies

$$
\begin{cases}z_{\tau}=z_{y y}, & (y, \tau) \in \mathbb{R}_{+} \times \mathbb{R}_{+}, \\ -z_{y}(0, \tau)=0, & \tau \in \mathbb{R}_{+} \\ z(y, 0)=v_{T}, & y \in \mathbb{R}_{+}\end{cases}
$$

Here we have used that, since $p_{21}<p_{11}-1$, then $p_{11}-p_{21}>0$, and that

$$
\lim _{n \rightarrow \infty}-n^{2\left(p_{11}-1\right)} t_{u}(n)=-\infty .
$$

We conclude that $z \equiv v_{T}$ for all $y$ and $\tau$.

Let

$$
\Phi(y, \tau)=\lim _{n \rightarrow \infty} w_{n}(y, \tau) .
$$

Passing to the limit in the equation we get

$$
\begin{cases}\Phi_{\tau}=\Phi_{y y}, & (y, \tau) \in \mathbb{R}_{+} \times \mathbb{R}, \\ -\Phi_{y}(0, \tau)=f_{1}^{11}(\Phi(0, \tau)) v_{T}^{p_{12}}, & \tau \in \mathbb{R} .\end{cases}
$$

This problem does not have uniqueness because it is invariant under time translations. In order to characterize the limit completely we need some extra information. This information comes from the behaviour of the component $u$ as $t \nearrow T$. Actually, from Lemma 4.1 we know that the blow-up profile is self-similar,

$$
\lim _{t \nearrow T}(T-t)^{\frac{1}{2\left(p_{11}-1\right)}} u\left(y(T-t)^{1 / 2}, t\right)=v_{T}^{\frac{p_{12}}{p_{11}-1}} \zeta(y),
$$


uniformly on compact intervals $0 \leq y \leq C$. The unique solution of (4.2) giving the asymptotic behaviour is the only one that coincides for $\tau$ negative and large enough with the self-similar function

$$
w(y, \tau)=v_{T}^{\frac{p_{12}}{p_{11}-1}}(-\tau)^{-\frac{1}{2\left(p_{11}-1\right)}} \zeta\left(y(-\tau)^{-1 / 2}\right),
$$

see [17] for the details.

Proof of Theorem 1.5. Let $z_{n}(y, \tau)=v_{n}\left(n^{-p_{21}} y, T+n^{-2 p_{21}} \tau\right)$. The function $z_{n}$ satisfies

$$
\begin{cases}\left(z_{n}\right)_{\tau}=\left(z_{n}\right)_{y y}, & (y, \tau) \in \mathbb{R}_{+} \times \mathbb{R}_{+}, \\ -\left(z_{n}\right)_{y}(0, \tau)=f_{n}^{22}\left(z_{n}(0, \tau)\right), & \tau \in \mathbb{R}_{+}, \\ z_{n}(y, 0)=v_{n}\left(n^{-p_{21}} y, T\right), & y \in \mathbb{R}_{+} .\end{cases}
$$

Since $z_{n}$ is uniformly bounded for $\tau$ in compact subsets of $[0, \widetilde{\tau})$ for some $\widetilde{\tau}>0$ and $v_{n}\left(n^{-p_{21}} y, T\right)$ goes to $v_{T}$ as $n \rightarrow \infty$,

$$
\lim _{n \rightarrow \infty} z_{n}(y, \tau)=\lim _{n \rightarrow \infty} v_{n}\left(n^{-p_{21}} y, T+n^{-2 p_{21}} \tau\right)=\Psi(y, \tau),
$$

where $\Psi$ is the solution of the heat equation with initial datum $\Psi(y, 0)=v_{T}$ and boundary flux $-\Psi_{y}(0, \tau)=\Psi^{p_{22}}(0, \tau)$. This limit $\Psi$ blows up in a finite time $\tau_{0}$ when $p_{22}>1$. With this fact in mind one can extend the convergence to compact subsets of $\overline{\mathbb{R}_{+}} \times\left[0, \tau_{0}\right)$.

\section{Avalanche}

Proof of Theorem 1.6. Let $z_{n}(x, t)=n^{-\gamma_{2}} v_{n}(x, t)$. For times $\tau>t_{u}(n)$, the truncation for $u_{n}$ has already taken place. Hence $z_{n}$ is a solution to the heat equation in $\mathbb{R}_{+} \times(\tau, \infty)$ with initial data $z_{n}(x, \tau)=n^{-\gamma_{2}} v_{n}(x, \tau)$, and boundary flux

$$
-\left(z_{n}\right)_{x}(0, t)=n^{p_{21}-\gamma_{2}} f_{n}^{22}\left(v_{n}(0, t)\right), \quad t \in(\tau, \infty) .
$$

The idea is to choose $\gamma_{2}$ so that in the limit we get a nontrivial boundary flux condition. This choice depends on the value of $f_{n}^{22}\left(v_{n}(0, t)\right)$; i.e. on the behaviour of $t_{v}(n)$ as $n$ tends to infinity.

$p_{21}+p_{22}<1 \quad$ In this range of parameters $t_{v}(n) \rightarrow \infty$, see Theorem 1.2. Hence, for any fixed time $\bar{t}>T$ and $n$ large, $t_{v}(n)>\bar{t}$. Thus, $v_{n}$ never reaches level $n$ and equation (5.1) becomes, taking $\tau=t_{u}(n)$ and $\gamma_{2}=\frac{p_{21}}{1-p_{22}}$,

$$
-\left(z_{n}\right)_{x}(0, t)=n^{p_{21}-\gamma_{2}+\gamma_{2} p_{22}} z_{n}^{p_{22}}(0, t)=z_{n}^{p_{22}}(0, t), \quad t \in\left(t_{u}(n), \bar{t}\right) .
$$

Using a comparison argument we have, for $t \in\left[t_{u}(n), \bar{t}\right]$ and $x \in \overline{\mathbb{R}_{+}}$,

$$
\psi_{n}(x, t) \leq z_{n}(x, t) \leq \varepsilon+\psi_{n}(x, t),
$$


where $\psi_{n}$ is the unique positive solution of the heat equation in $\mathbb{R}_{+} \times\left(t_{u}(n), \infty\right)$ with boundary flux $-\left(\psi_{n}\right)_{x}(0, t)=\psi_{n}^{p_{22}}(0, t)$ and initial datum $\psi_{n}\left(x, t_{u}(n)\right)=0$. Letting first $n \rightarrow \infty$ and then $\varepsilon \rightarrow 0, z_{n}$ converges uniformly on compact subsets of $[0, L] \times(T, \infty)$ (notice that $\bar{t}$ is arbitrary) to $\psi$, the unique positive solution to

$$
\begin{cases}\psi_{t}=\psi_{x x}, & (x, t) \in \mathbb{R}_{+} \times(T, \infty), \\ \psi(x, T)=0, & x \in \mathbb{R}_{+},\end{cases}
$$

with boundary flux

$$
-\psi_{x}(0, t)=\psi^{p_{22}}(0, t), \quad t \in(T, \infty) .
$$

This function has a self similar form $\psi(x, t)=(t-T)^{1 /\left(2\left(p_{22}-1\right)\right)} \phi\left(x(t-T)^{-1 / 2}\right)$, see [5].

$p_{21}+p_{22}=1 \quad$ In this case $t_{v}(n) \sim T$, see Theorem 1.2, and we cannot determine if the truncation takes place or not before $\bar{t}$. In any case, we take $\gamma_{2}=1$, so that (5.1) is independent of $n$. Hence the limit function $\psi(x, t)$ is now a positive solution to (5.2), but with boundary flux given by

$$
-\psi_{x}(0, t)=f_{1}^{22}(\psi(0, t)), \quad t \in(T, \infty) .
$$

Since $p_{22}<1$ this problem has a positive solution (that can be obtained as in [5]). As before $\psi$ has a self-similar form until the time $\tilde{t}$ where it reaches level one, $\psi(0, \tilde{t})=1$. From that time it behaves like the solution of the heat equation with boundary flux equal to one and initial datum $\psi(x, \tilde{t})$.

$p_{21}+p_{22}>1 \quad$ Let $\bar{t}>T$ be a fixed time. Since $t_{v}(n) \rightarrow T$, for large values of $n$, we haver that $t_{v}(n)<\bar{t}$, the truncation for $v_{n}$ takes place, and (5.1) becomes

$$
-\left(z_{n}\right)_{x}(0, t)=n^{p_{21}-\gamma_{2}+p_{22}}=1, \quad t \in\left(t_{v}(n), \bar{t}\right),
$$

if $\tau=t_{v}(n)$ and $\gamma_{2}=p_{21}+p_{22}$. This choice of $\gamma_{2}$ implies that $z\left(x, t_{v}(n)\right) \leq n^{1-\gamma_{2}} \rightarrow 0$ as $n \rightarrow \infty$. Using a comparison argument as before, we have that $z_{n}$ converges uniformly on compact subsets of $[0, \infty) \times(T, \infty)$ to $\psi$, the unique positive solution of $(5.2)$ with boundary data

$$
-\psi_{x}(0, t)=1, \quad t \in(T, \infty) .
$$

Again in this case $\psi$ has a self-similar structure, $\psi(x, t)=(t-T)^{1 / 2} \phi\left(x(t-T)^{-1 / 2}\right)$. The profile $\phi$ can be computed explicitly.

To conclude the proof we have to deal with the limit of $u_{n}(x, t)$. To this aim we define $w_{n}(x, t)=n^{-\gamma_{1}} u_{n}(x, t)$, which is a solution to

$$
\begin{cases}\left(w_{n}\right)_{t}=\left(w_{n}\right)_{x x}, & (x, t) \in \mathbb{R}_{+} \times\left(t_{u}(n), \infty\right), \\ -\left(w_{n}\right)_{x}(0, t)=n^{p_{11}-\gamma_{1}} f_{n}^{12}\left(n^{\gamma_{2}} z_{n}(0, t)\right), & t \in\left(t_{u}(n), \infty\right), \\ w_{n}\left(x, t_{u}(n)\right)=n^{-\gamma_{1}} u_{n}\left(x, t_{u}(n)\right) \leq n^{1-\gamma_{1}}, & x \in \mathbb{R}_{+},\end{cases}
$$

with $\gamma_{1}$ a constant. As we already know that $z_{n}(0, t) \rightarrow \psi(0, t)$ uniformly on $t \in(T, \infty)$, we pass to the limit and obtain that there exists a nontrivial profile $\varphi$ such that 
$w_{n}(x, t) \rightarrow \varphi(x, t)$, as $n \rightarrow \infty$. This limit profile is a solution to the heat equation in $\mathbb{R}_{+} \times(T, \infty)$ and if $\gamma_{1}>1$ (which will be shown to be true) the initial datum becomes $\varphi(x, T)=0$. The constant $\gamma_{1}$ is chosen so that the limit boundary condition

$$
-\varphi_{x}(0, t)=n^{p_{11}-\gamma_{1}} f_{n}^{12}\left(n^{\gamma_{2}} \psi(0, t)\right)=n^{p_{11}-\gamma_{1}+\gamma_{2} p_{12}} f_{n^{1-\gamma_{2}}}^{12}(\psi(0, t))
$$

is independent of $n$.

$p_{21}+p_{22}<1 \quad$ Since $\psi$ is bounded, we have that $f_{n^{1-\gamma_{2}}}^{12}(\psi(0, t))=\psi^{p_{12}}(0, t)$, for $n$ large enough. Hence, taking $\gamma_{1}=p_{11}+\gamma_{2} p_{12}$ we get $-\varphi_{x}(0, t)=\psi^{p_{12}}(0, t)$.

$p_{21}+p_{22}=1 \quad$ We have $\gamma_{2}=1$, therefore the boundary condition (5.3) becomes $-\varphi_{x}(0, t)=f_{1}^{12}(\psi(0, t))$ if $\gamma_{1}=p_{11}+p_{12}$.

$p_{21}+p_{22}>1$ Since $\gamma_{2}>1$, we take $\gamma_{1}=p_{11}+p_{12}$ and (5.3) turns out to be $-\varphi_{x}(0, t)=1$.

\section{Acknowledgement}

Cristina Brändle and Fernando Quirós partially supported by Project MTM2005-08760-C02-01, Project CCG06-UAM/ESP-0302 (Spain) and ESF Programme "Global and geometric aspects of nonlinear partial differential equations". Julio D. Rossi partially supported by ANPCyT PICT 5009, UBA X066 and CONICET (Argentina).

\section{References}

[1] Baras, P.; Cohen, L. Complete blow-up after $T_{\max }$ for the solution of a semilinear heat equation. J. Funct. Anal. 71 (1987), no. 1, 142-174.

[2] Brändle, C.; Quirós, F.; Rossi, J. D. A complete classification of simultaneous blow-up rates. Appl. Math. Lett. 19 (2006), no. 7, 607-611.

[3] Brändle, C.; Quirós, F.; Rossi, J. D. Non-simultaneous blow-up for a quasilinear parabolic system with reaction at the boundary. Commun. Pure Appl. Anal. 4 (2005), no. 3, 523-526.

[4] Chlebík, M.; Fila, M. Some recent results on blow-up on the boundary for the heat equation. Evolution equations: existence, regularity and singularities (Warsaw, 1998), 61-71. Banach Center Publ., vol. 52. Polish Acad. Sci. Warsaw. 2000.

[5] Cortazar, C.; Elgueta, M.; Rossi, J. D. Uniqueness and Nonuniqueness for a System of Heat Equations with nontrivial coupling at the boundary. Nonlinear Analysis TM\&A. 37 (1999), no. 2, 257-267.

[6] Fila, M.; Filo, J. Blow-up on the boundary: a survey. Singularities and differential equations (Warsaw, 1993) 67-78. Banach Center Publ., vol. 33. Polish Acad. Sci. Warsaw. 1996.

[7] Fila, M.; Guo, J-S. Complete blow-up and incomplete quenching for the heat equation with a nonlinear boundary condition. Nonlinear Anal. 48 (2002), no. 7, Ser. A: Theory Methods, 995-1002.

[8] Fila, M.; Quittner, P. The blow-up rate for the heat equation with a nonlinear boundary condition. Math. Meth. Appl. Sci. 14 (1991), 197-205. 
[9] Galaktionov, V. A.; Vázquez, J. L. Necessary and sufficient conditions for complete blow-up and extinction for one-dimensional quasilinear heat equations. Arch. Rational Mech. Anal. 129 (1995), no. 3, 225-244.

[10] Galaktionov, V. A.; Vázquez, J. L. Continuation of blowup solutions of nonlinear heat equations in several space dimensions. Comm. Pure Appl. Math. 50 (1997), no. 1, 1-67.

[11] Galaktionov, V. A.; Vázquez, J. L. The problem of blow-up in nonlinear parabolic equations. Current developments in partial differential equations (Temuco, 1999). Discrete Contin. Dyn. Syst. 8 (2002), no. 2, 399-433.

[12] Lacey, A. A.; Tzanetis, D. Complete blow-up for a semilinear diffusion equation with a sufficiently large initial condition. IMA J. Appl. Math. 41 (1988), no. 3, 207-215.

[13] Martel, Y. Complete blow up and global behaviour of solutions of $u_{t}-\Delta u=g(u)$. Ann. Inst. H. Poincaré. Anal. Non Linéaire 15 (1998), no. 6, 687-723.

[14] Pinasco, J. P.; Rossi, J. D. Simultaneous versus non-simultaneous blow-up. New Zealand J. Math. 29 (2000), no. 1, 55-59.

[15] Quirós, F.; Rossi, J. D. Non-simultaneous blow-up in a semilinear parabolic system. Z. Angew. Math. Phys. 52 (2001), no. 2, 342-346.

[16] Quirós, F.; Rossi, J. D. Non-simultaneous blow-up in a nonlinear parabolic system. Adv. Nonlinear Stud. 3 (2003), no. 3, 397-418.

[17] Quirós, F.; Rossi, J. D.; Vázquez, J. L. Complete blow-up and thermal avalanche for heat equations with nonlinear boundary conditions. Comm. Partial Differential Equations 27 (2002), no. 1-2, 395-424.

[18] Quirós, F.; Rossi, J. D.; Vázquez, J. L. Thermal avalanche for blowup solutions of semilinear heat equations. Comm. Pure Appl. Math. 57 (2004), no. 1, 59-98.

[19] Samarskii, A. A.; Galaktionov, V. A.; Kurdyumov, S. P.; Mikhailov, A. P. "Blow-up in quasilinear parabolic equations". de Gruyter Expositions in Mathematics, vol. 19. Walter de Gruyter \& Co. Berlin. 1995. Translated from the 1987 Russian original by Michael Grinfeld and revised by the authors.

[20] Souplet, P.; Tayachi, S. Optimal condition for non-simultaneous blow-up in a reactiondiffusion system. J. Math. Soc. Japan 56 (2004), no. 2, 571-584. 